\title{
Noninvasive diagnosis of pediatric nonalcoholic fatty liver disease
}

Hye Ran Yang, MD, PhD

Department of Pediatrics, Seoul National University Bundang Hospital, Seoul National University College of Medicine, Seongnam, Korea

Because nonalcoholic steatohepatitis can progress towards cirrhosis even in children, early detection of hepatic fibrosis and accurate diagnosis of nonalcoholic fatty liver disease (NAFLD) are important. Although liver biopsy is regarded as the gold standard of diagnosis, its clinical application is somewhat limited in children due to its invasiveness. Noninvasive diagnostic methods, including imaging studies, biomarkers of inflammation, oxidative stress, hepatic apoptosis, hepatic fibrosis, and noninvasive hepatic fibrosis scores have recently been developed for diagnosing the spectrum of NAFLD, particularly the severity of hepatic fibrosis. Although data and validation are still lacking for these noninvasive modalities in the pediatric population, these methods may be applicable for pediatric NAFLD. Therefore, noninvasive imaging studies, biomarkers, and hepatic fibrosis scoring systems may be useful in the detection of hepatic steatosis and the prediction of hepatic fibrosis, even in children with NAFLD.

Key words: Nonalcoholic fatty liver disease, Fibrosis, Diagnosis, Obesity, Child

\section{Introduction}

Nonalcoholic fatty liver disease (NAFLD) is a form of chronic liver disease with histopathologic features of typical alcohol-induced chronic liver disease that develops in individuals who do not consume alcohol excessively ${ }^{11}$. Since Ludwig et al. ${ }^{2)}$ described nonalcoholic steatohepatitis (NASH) in 1980, there have been remarkable advances in diagnosing NAFLD. The disease spectrum of NAFLD ranges from simple steatosis to NASH, nonalcoholic hepatic fibrosis, and nonalcoholic cirrhosis, and NASH progresses towards cirrhosis, even in children ${ }^{3-5)}$. Therefore, early detection of hepatic fibrosis and accurate diagnosis of NAFLD are essential, and NASH, fibrosis, and cirrhosis should be distinguished from benign simple steatosis in obese children suspected to have NAFLD. Typically, the diagnosis of NASH is based on clinical exclusion of alcohol or drug intake, followed by serologic exclusion of other underlying liver diseases, and, finally, percutaneous liver biopsy. Percutaneous liver biopsy is regarded as the gold standard for diagnosing NAFLD, even in children, because a defınite NAFLD diagnosis requires histological data such as macrovesicular steatosis, necroinflammation, and hepatic fibrosis ${ }^{6}$.

As Roberts ${ }^{7}$ suggested in 2007, candidate criteria for liver biopsy in suspected pediatric NAFLD include factors such as an age of less than 10 years, hepatosplenomegaly, highly increased serum aminotransferase level, severe insulin resistance, comorbid chronic liver diseases, and family history. However, the use of liver biopsy is limited in the pediatric population because of its invasiveness and the fact that the histologic features of pediatric NASH are somewhat different from those of adult $\mathrm{NASH}^{8,99}$.
Corresponding author: Hye Ran Yang, MD, PhD Department of Pediatrics, Seoul National University Bundang Hospital, Seoul National University College of Medicine, 82 Gumi-ro 173beon-gil, Bundang-gu, Seongnam 463-707, Korea

Tel: $+82-31-787-7285$

Fax: +82-31-787-4054

E-mail: hryang@snubh.org

Received: 16 September 2012

Accepted: 2 October 2012

Copyright (c) 2013 by The Korean Pediatric Society

This is an open-access article distributed under the terms of the Creative Commons Attribution NonCommercial License (http://creativecommons.org/ licenses/by-nc/3.0/) which permits unrestricted noncommercial use, distribution, and reproduction in any medium, provided the original work is properly cited. 
Recently, noninvasive diagnostic methods, including imaging studies for detecting steatosis or fibrosis, and biomarkers of oxidative stress, inflammation, hepatocyte apoptosis, and hepatic fibrosis have been introduced to diagnose the spectrum of NAFLD ${ }^{10,11)}$. Additionally, noninvasive markers of hepatic fibrosis and noninvasive hepatic fibrosis scores have also been developed and applied for evaluating the severity of hepatic fibrosis, mainly in adults ${ }^{12-14)}$.

To date, only a limited number of studies have examined noninvasive biochemical markers of hepatic fibrosis for pediatric NAFLD ${ }^{15-17)}$ and only a few studies have been performed on previously suggested fibrosis scores or NAFLD-related hepatic fibrosis scores in children ${ }^{18-20)}$. Although data and validation studies in the pediatric population are still lacking, these noninvasive modalities can also be applied for pediatric NAFLD.

In this review, noninvasive clinical and laboratory parameters as possible clues of NAFLD, noninvasive imaging studies for detecting hepatic steatosis (and possibly fibrosis), newly developed noninvasive biomarkers for diagnosing NAFLD, and noninvasive hepatic fibrosis scores indicating the severity of hepatic fibrosis will be discussed, with a primary focus on pediatric NAFLD.

\section{Clinical and laboratory clues to NAFLD in obese children}

Most cases of NAFLD in obese children and adolescents are incidentally detected by health checkups at schools or hospitals. Most patients with NAFLD are asymptomatic, except for nonspecific clinical symptoms or signs such as easy fatigability and right upper quadrant abdominal discomfort or hepatomegaly and acanthosis nigricans in some patients ${ }^{21,22)}$. There are no clinical features definitely indicative of NAFLD in obese children. However, some clinical features such as obstructive sleep apnea or acanthosis nigricans may be significantly correlated with NAFLD ${ }^{23)}$.

Increased levels of serum aspartate aminotransferase (AST) and alanine aminotransferase (ALT) in obese patients are wellknown predictors of NAFLD, particularly NASH, although serum aminotransferase levels sometimes remain within a normal range despite the presence of NAFLD ${ }^{21)}$. Mildly elevated levels of alkaline phosphatase or gamma glutamyl transpeptidase (GGT) have been associated with NAFLD ${ }^{21)}$. However, the levels of these enzymes do not reflect the severity of steatosis or hepatic fibrosis in patients with NAFLD.

According to a recent study on pediatric $\mathrm{NASH}^{24)}$, most NAFLD patients were obese children with a body mass index (BMI) higher than the 95th percentile, and other demographic features were not significantly different among the 3 groups examined (no NASH, adult-type NASH, and definite NASH), although age and ethnicity differed between pediatric-type NASH and definite NASH. Increased levels of serum AST and GGT were significant predictors of NAFLD among the 3 groups, as were age, prothrombin time, and insulin resistance between 2 groups $^{24)}$. Additionally, increased serum AST levels, white blood cell count, and decreased hematocrit levels were found to be significant predictors of moderate fibrosis in that study ${ }^{24)}$. In contrast, a multicenter study in 2009 showed that none of the clinical features, including BMI or comorbid diseases, were significantly correlated with the stage of fibrosis; laboratory markers were also not correlated, except for serum AST levels ${ }^{25}$. The serum AST levels were correlated with the stage of fibrosis and could be used to distinguish significant fibrosis from no or mild fibrosis ${ }^{25)}$. However, in this study, the authors concluded that AST is a good predictor of severe fibrosis in children with NAFLD, based on the low value (0.63) obtained for the area under the receiver operating curve (AUROC) ${ }^{25}$. In our recent studies, no single clinical or laboratory parameters reflected the presence or severity of hepatic fibrosis in children with NAFLD $^{18)}$, except for BMI, which was significantly different among children with simple steatosis, type 1 adult NASH, and type 2 pediatric $\mathrm{NASH}^{9)}$.

Thus, noninvasive tools beyond clinical or laboratory markers are required to diagnose NAFLD in children, such as noninvasive imaging studies, biomarkers, or hepatic fibrosis scores.

\section{Noninvasive imaging studies for pediatric NAFLD}

Noninvasive imaging modalities for NAFLD include abdominal ultrasonography (USG), abdominal computed tomography (CT), magnetic resonance imaging (MRI), fibroscan (hepatic elastography), magnetic resonance (MR) elastography, and MR spectroscopy ${ }^{11)}$.

Among noninvasive imaging techniques, abdominal USG has some advantages because it is a safe and economic modality showing a wide range of sensitivity and specificity in screening for the presence of hepatic steatosis in patients with NAFLD. Thus, abdominal USG is frequently used to detect the presence of fatty liver and to evaluate the degree of hepatic steatosis in the clinical field, although the diagnostic accuracy of liver USG in children has not yet been determined ${ }^{26)}$. Ultrasonographic criteria for assessing hepatic steatosis include hepatorenal echo contrast, liver brightness, intrahepatic vessel blurring, and visualization of the liver parenchyma and diaphragm ${ }^{11}$. According to a recent study examining the clinical utility of ultrasonographic quantification of hepatic steatosis, liver USG is a useful tool for quantifying hepatic steatosis in children suspected to have NAFLD, because the degree of steatosis on 
USG was strongly correlated with the steatosis grade obtained on liver histopathological analysis ${ }^{26)}$.

Unenhanced abdominal CT is useful for detecting the presence of fatty liver and visceral adiposity and sufficiently sensitive to quantify hepatic steatosis ${ }^{27)}$. The diagnostic accuracy of abdominal CT was relatively high in cases where hepatic steatosis was found to be present in more than 30\% of the liver when a liver-spleen house field unit ratio was applied ${ }^{28)}$. However, CT is not recommended for the pediatric population due to the radiation hazard.

Compared with abdominal CT, MRI is a highly accurate imaging modality that is useful for identifying and quantifying hepatic steatosis safely, even in children, without radiation hazard $^{29)}$. The disadvantages of using MRI for diagnosing NAFLD include high costs and limitations in patients with claustrophobia, metallic devices, or iron overload ${ }^{30}$.

Clinically, abdominal USG, CT, and MRI have some limitations because these methods cannot accurately differentiate between simple steatosis and NASH and cannot confirm the presence or stages of hepatic fibrosis ${ }^{11}$. Recently introduced methods such as fibroscan, MR elastography, and MR spectroscopy are promising modalities expected to overcome the limitations of conventional imaging studies in detecting hepatic fibrosis or steatosis.

According to recent studies, fibroscan (transient elastography) using an ultrasonographic technique can be applied to noninvasively detect liver fibrosis by measuring liver stiffness in both adults and children, although this method has some disadvantages, including decreased performance with increase in BMI and also expensive equipment ${ }^{31,32)}$. MR elastography, introduced for the assessment of hepatic fibrosis in adult patients with NAFLD, is a noninvasive imaging technique that can also be applied in children, despite some limitations ${ }^{33,34)}$. Additionally, 1H-MR spectroscopy may be an alternative noninvasive method for identifying and measuring hepatic fat content in patients with NAFLD ${ }^{35}$.

Regarding the noninvasive diagnosis of pediatric NAFLD by using imaging techniques, in 2012, the Hepatology Committee of European Society for Pediatric Gastroenterology, Hepatology, and Nutrition (ESPGHAN) concurred that most studies examining various imaging methods had been performed with adult NAFLD patients and that only a few studies had been performed for a large group of pediatric NAFLD patients ${ }^{36}$. The committee reached a consensus in that abdominal USG is a safe method with a limited ability to quantify steatosis or fibrosis and that abdominal MRI is a very expensive, rapid, and reproducible method for measuring steatosis and fibrosis after some modifications ${ }^{36}$. Fibroscan is not yet universally recommended for pediatric NAFLD ${ }^{36}$.

\section{Noninvasive biomarkers of NAFLD in children}

The ideal biomarkers for NAFLD are those that would be simple and easy to measure, cost-effective, accurate in diagnosing NAFLD, capable of helping evaluate the stage of fibrosis and to monitor treatment response, and validated in a large or multicenter study ${ }^{10)}$. There are 4 major categories of noninvasive biomarkers that can be used to indicate NAFLD not only in adults but also in the pediatric population: hepatic inflammation, oxidative stress, hepatocyte apoptosis, and hepatic fibrosis $^{10,11,23,36)}$.

\section{Category I. Biomarkers of hepatic inflammation}

Beyond laboratory markers of systemic inflammation such as C-reactive protein (CRP) or high sensitive CRP, cytokines have been suggested as possible biomarkers of hepatic inflammation in both adults and children with $\mathrm{NASH}^{10,23,36)}$. Increased levels of serum tumor necrosis factor (TNF)- $\alpha$ and interleukin6 , which are proinflammatory cytokines, were noted in patients with NAFLD, and increased levels of leptin and decreased levels of adiponectin, which are adipocytokines, are also reported to be related to NAFLD development in adults and children.

Controversy exists regarding the elevation of serum TNF- $\alpha$ levels in children with NAFLD: one study reported a correlation between serum TNF- $\alpha$ levels and NAFLD activity scores (NASs) obtained on liver biopsy ${ }^{37)}$, whereas other studies, including our recent study, reported no significant differences in serum TNF- $\alpha$ levels among different NAFLD groups ${ }^{38}$. The serum concentration of leptin, an adipokine that is produced in adipose tissue and acts as a marker of insulin resistance, is also controversial in pediatric NAFLD. This is because only a few studies have reported a correlation between serum leptin levels and the histological features of NAFLD or higher serum leptin levels in children with NAFLD than in controls ${ }^{37,39)}$, whereas other studies have reported no significant difference in serum leptin levels ${ }^{40)}$.

Adiponectin is also an adipokine and acts as a marker of insulin sensitivity. In some studies on pediatric NAFLD, serum adiponectin levels were found to decrease in obese children with elevated serum ALT levels and in obese children with fatty liver confirmed using $\mathrm{USG}^{39,41}$. In our previous study on children with histopathologically confirmed NAFLD, serum adiponectin levels were lower in patients with NASH than in controls and patients with simple steatosis ${ }^{40)}$.

Recently, retinol-binding protein 4 (RBP4), which is an another adipocytokine associated with insulin resistance, was introduced as a possible noninvasive marker in children with NAFLD, indicating that circulating RBP4 is potentially related with the degree of liver damage in pediatric NAFLD ${ }^{5,422}$.

These data suggest that measuring various biomarkers of 
hepatic inflammation such as proinflammatory cytokines and adipocytokines may be useful for detecting NAFLD and for identifying NASH among the spectrum of NAFLD in children. However, to date, there is a lack of data to support the widespread clinical application of these cytokines for diagnosing NASH in children.

\section{Category II. Biomarkers of oxidative stress}

According to a 2-hit hypothesis as a possible mechanism for the pathogenesis of NAFLD ${ }^{42)}$, the first hit for developing NAFLD is the accumulation of macrovesicular fat in the liver, causing hepatic steatosis, and the second hit mainly includes oxidative stress producing excessive reactive oxidative species and free radicals via mitochondrial and peroxisomal pathways in the liver, thereby causing hepatocyte injury, inflammation, and fibrosis. Thus, measuring biomarkers of oxidative stress is expected to reflect NAFLD status; these biomarkers include lipid peroxidation products, antioxidant glutathione, or protein oxidation products ${ }^{10)}$.

According to a recent study by Bell et al. ${ }^{43)}$, hepatic malondialdehyde levels for hepatic lipid peroxidation were higher in children with biopsy-confirmed NAFLD than in controls or children with hepatitis $\mathrm{C}$ infection, although there was no significant difference between hepatic cytochrome P450 2E1 protein levels in both groups. In a 2008 study, protein glutathionylation, occurring as a response to oxidative stress, was found to have increased in the liver tissue of children with NAFLD; furthermore, it was found to be correlated with NASH and fibrosis ${ }^{44)}$. Nobili et al. ${ }^{45)}$ reported that oxidative stress parameters, including the levels of serum protein carbonyls, hepatic 8-hydroxy-2-deoxyguanosine, and circulating antimalondialdehyde adduct human serum albumin (anti-MDAHSA) immunoglobulin G antibody, were elevated in children with NAFLD and that anti-MDA-HSA levels correlated with NAS, reflecting the severity of NASH on liver biopsy. The results of these studies indicate that biomarkers of oxidative stress may be promising parameters for evaluating pediatric NAFLD, although these parameters remain controversial for adults ${ }^{46)}$. Further studies on clinically available serum biomarkers of oxidative stress are required.

\section{Category III. Biomarkers of apoptosis}

Recently, cytokeratin-18 (CK-18), which is a marker of hepatocyte apoptosis, has been regarded as the most promising biomarker for NASH. Some studies in adults and children have reported the role of CK-18 in differentiating NASH from benign simple steatosis ${ }^{47-50)}$. CK-18 levels were significantly higher in children with suspected NAFLD than in controls, indicating that CK-18 is the best single independent predictor of NAFLD in children $^{49)}$. Even in biopsy-proven pediatric NAFLD, the level of the CK-18 fragment was higher in children with NAFLD than in controls ${ }^{50)}$. Moreover, the CK-18 levels were higher in children with NASH than in those with simple steatosis, and CK18 distinguished significant fibrosis from no or mild fibrosis in children $^{50)}$. Therefore, CK-18, a biomarker of apoptosis, is a good predictor of NASH and hepatic fibrosis, even in the pediatric population.

\section{Category IV. Biomarkers of hepatic fibrosis}

Ideal hepatic fibrosis biomarkers would be those that are liverspecific and whose tests are easy to perform; the levels of these biomarkers would be minimally altered by biliary or urinary excretion or metabolism and would be reflective of hepatic fibrosis from any chronic liver injury, sufficiently sensitive to stratify stages of hepatic fibrosis, associated with progression or regression of hepatic fibrosis, and able to predict clinical outcome $^{13)}$. Biomarkers of hepatic fibrosis or fibrogenesis have been suggested, including tumor growth factor- $\beta$ (TGF- $\beta$ ), hyaluronic acid, laminin, type IV collagen, and other extracellular matrix components, although there are some limitations to these molecules ${ }^{10)}$.

Among these biomarkers, serum TGF- $\beta$ levels showed no significant relationship with stages of fibrosis according to histological results obtained for adults ${ }^{51)}$.

A previous study on tissue inhibitor of metalloproteinase-1 (TIMP-1) and hyaluronic acid showed that both serum TIMP-1 and hyaluronic acid levels were significantly different in adult patients with $\mathrm{NASH}^{52)}$. In a pediatric study, hyaluronic acid levels of $\geq 1,200 \mathrm{ng} / \mathrm{mL}$ ruled out fibrosis (stage 0 ) and levels that were $\geq 2,100 \mathrm{ng} / \mathrm{mL}$ significantly distinguished advanced fibrosis (stage 2 or more) in children, indicating that hyaluronic acid may be a good predictor of hepatic fibrosis not only in adults but also in children ${ }^{16)}$.

Type IV collagen has been investigated only in adult patients with NASH and the results of a study suggested a weak but significant correlation between type IV collagen levels and stages of fibrosis; in this study, higher type IV collagen levels were seen in adults with advanced fibrosis (stage 3 or more) than in those with mild fibrosis ${ }^{53)}$. However, not much data are available regarding the application of type IV collagen for assessing hepatic fibrosis stages.

Thus, further studies are required to develop a single biomarker of hepatic fibrosis that can detect the presence of fibrosis and discriminate advanced fibrosis from no or mild fibrosis.

\section{Noninvasive hepatic fibrosis scores of NAFLD in children}

To date, a variety of noninvasive hepatic fibrosis scores, 
involving of one or more clinical parameters and laboratory markers, have been developed and applied in adults with NAFLD to differentiate NASH from benign simple steatosis, particularly in terms of the presence of hepatic fibrosis ${ }^{14}$.

The AST/ALT ratio is a commonly used indicator of hepatic fibrosis because it is simple and easy to calculate; however, the diagnostic accuracy of an AST/ALT ratio $>1$ is very low in children with NAFLD, limiting its routine use as a hepatic fibrosis score $^{18,24)}$.

The AST-platelet ratio index (APRI), which includes AST and platelet count, has primarily been used to predict hepatic fibrosis in various chronic liver diseases in adults and children ${ }^{54,55)}$. In our previous study on pediatric NAFLD, APRI was an excellent predictor of significant hepatic fibrosis (stage 2 or more) in children with biopsy-proven NASH ${ }^{18)}$.

FibroTest, which involves total bilirubin, GGT, $\alpha 2$-macroglobulin, apolipoprotein A1, and haptoglobin levels, may be a good predictor of advanced fibrosis, but it has been evaluated only in adults with NAFLD and not in children ${ }^{56)}$.

The FIB-4 score, which involves age, AST, ALT levels, and platelet count, has been applied in adults with NAFLD and has been found to be a better noninvasive method of scoring fibrosis than other scores ${ }^{57)}$. In our recent study published in 2012, FIB-4 was first evaluated in children with biopsy-confirmed NAFLD and showed the highest diagnostic accuracy among various noninvasive hepatic fibrosis scores in differentiating significant fibrosis (stage 2 or more) from no or mild fibrosis in pediatric NAFLD ${ }^{18)}$.

The enhanced liver fibrosis (ELF) test, involving hyaluronic acid, amino-terminal propeptide of type III collagen, and TIMP-1, was found to be a good predictor in adult patients with chronic liver disease ${ }^{58)}$, and was then also applied to children with NAFLD in order to predict hepatic fibrosis ${ }^{19)}$. The ELF test can differentiate significant fibrosis (stage 3 or more) from no or mild fibrosis, with an AUROC of 0.99 for a cutoff of 10.51 in children ${ }^{19)}$.

The pediatric NAFLD fibrosis index (PNFI) is the only noninvasive hepatic fibrosis scoring system developed for children with NAFLD and involves age, waist circumference, and serum triglyceride levels ${ }^{20)}$. According to previous studies, PNFI is useful for differentiating children with hepatic fibrosis from those without fibrosis with an AUROC of 0.85 and 0.761, respectively $^{20,59)}$. On combining the PNFI and ELF tests, the AUROC for predicting hepatic fibrosis increased up to 0.944 in children ${ }^{59)}$.

\section{Conclusion}

Liver biopsy is regarded as the gold standard of diagnosis of
NAFLD. However, the use of liver biopsy is clinically limited in children due to its invasiveness. Noninvasive diagnostic methods such as imaging studies, biomarkers of inflammation, oxidative stress, hepatic apoptosis, and hepatic fibrosis, and noninvasive hepatic fibrosis scores may be useful for overcoming these disadvantages.

Although these noninvasive methods have not been widely used or validated in pediatric NAFLD, some of them may be applicable for detecting hepatic steatosis and predicting hepatic fibrosis even in children with NAFLD.

\section{References}

1. Clark JM, Brancati FL, Diehl AM. Nonalcoholic fatty liver disease. Gastroenterology 2002;122:1649-57.

2. Ludwig J, Viggiano TR, McGill DB, Oh BJ. Nonalcoholic steatohepatitis: Mayo Clinic experiences with a hitherto unnamed disease. Mayo Clin Proc 1980;55:434-8.

3. Powell EE, Cooksley WG, Hanson R, Searle J, Halliday JW, Powell LW. The natural history of nonalcoholic steatohepatitis: a followup study of forty-two patients for up to 21 years. Hepatology 1990;11:74-80.

4. Molleston JP, White F, Teckman J, Fitzgerald JF. Obese children with steatohepatitis can develop cirrhosis in childhood. Am J Gastroenterol 2002;97:2460-2.

5. Feldstein AE, Charatcharoenwitthaya P, Treeprasertsuk S, Benson JT, Enders FB, Angulo P. The natural history of non-alcoholic fatty liver disease in children: a follow-up study for up to 20 years. Gut 2009;58:1538-44.

6. Takahashi Y, Fukusato T. Pediatric nonalcoholic fatty liver disease: overview with emphasis on histology. World J Gastroenterol 2010; 16:5280-5.

7. Roberts EA. Pediatric nonalcoholic fatty liver disease (NAFLD): a "growing" problem? J Hepatol 2007;46:1133-42.

8. Schwimmer JB, Behling C, Newbury R, Deutsch R, Nievergelt C, Schork NJ, et al. Histopathology of pediatric nonalcoholic fatty liver disease. Hepatology 2005;42:641-9.

9. Ko JS, Yoon JM, Yang HR, Myung JK, Kim H, Kang GH, et al. Clinical and histological features of nonalcoholic fatty liver disease in children. Dig Dis Sci 2009;54:2225-30.

10. Wieckowska A, McCullough AJ, Feldstein AE. Noninvasive diagnosis and monitoring of nonalcoholic steatohepatitis: present and future. Hepatology 2007;46:582-9.

11. Torres DM, Harrison SA. Diagnosis and therapy of nonalcoholic steatohepatitis. Gastroenterology 2008;134:1682-98.

12. Guha IN, Parkes J, Roderick PR, Harris S, Rosenberg WM. Noninvasive markers associated with liver fibrosis in non-alcoholic fatty liver disease. Gut 2006;55:1650-60.

13. Manning DS, Afdhal NH. Diagnosis and quantitation of fibrosis. Gastroenterology 2008;134:1670-81.

14. Shiha G, Sarin SK, Ibrahim AE, Omata M, Kumar A, Lesmana LA, et al. Liver fibrosis: consensus recommendations of the Asian Pacific Association for the Study of the Liver (APASL). Hepatol Int 2009;3:323-33.

15. Nobili V, Alkhouri N, Alisi A, Ottino S, Lopez R, Manco M, et al. Retinol-binding protein 4: a promising circulating marker of liver damage in pediatric nonalcoholic fatty liver disease. Clin Gast- 
roenterol Hepatol 2009;7:575-9.

16. Nobili V, Alisi A, Torre G, De Vito R, Pietrobattista A, Morino G, et al. Hyaluronic acid predicts hepatic fibrosis in children with nonalcoholic fatty liver disease. Transl Res 2010;156:229-34.

17. Iacobellis A, Marcellini M, Andriulli A, Perri F, Leandro G, Devito $\mathrm{R}$, et al. Non invasive evaluation of liver fibrosis in paediatric patients with nonalcoholic steatohepatitis. World J Gastroenterol 2006;12:7821-5.

18. Yang HR, Kim HR, Kim MJ, Ko JS, Seo JK. Noninvasive parameters and hepatic fibrosis scores in children with nonalcoholic fatty liver disease. World J Gastroenterol 2012;18:1525-30.

19. Nobili V, Parkes J, Bottazzo G, Marcellini M, Cross R, Newman D, et al. Performance of ELF serum markers in predicting fibrosis stage in pediatric non-alcoholic fatty liver disease. Gastroenterology 2009;136:160-7.

20. Nobili V, Alisi A, Vania A, Tiribelli C, Pietrobattista A, Bedogni G. The pediatric NAFLD fibrosis index: a predictor of liver fibrosis in children with non-alcoholic fatty liver disease. BMC Med 2009;7:21.

21. Papandreou D, Rousso I, Mavromichalis I. Update on non-alcoholic fatty liver disease in children. Clin Nutr 2007;26:409-15.

22. Schwimmer JB, Deutsch R, Rauch JB, Behling C, Newbury R, Lavine JE. Obesity, insulin resistance, and other clinicopathological correlates of pediatric nonalcoholic fatty liver disease. J Pediatr 2003;143:500-5.

23. Loomba R, Sirlin CB, Schwimmer JB, Lavine JE. Advances in pediatric nonalcoholic fatty liver disease. Hepatology 2009;50: 1282-93.

24. Patton HM, Lavine JE, Van Natta ML, Schwimmer JB, Kleiner D, Molleston J, et al. Clinical correlates of histopathology in pediatric nonalcoholic steatohepatitis. Gastroenterology 2008; 135:1961-71.

25. Carter-Kent C, Yerian LM, Brunt EM, Angulo P, Kohli R, Ling SC, et al. Nonalcoholic steatohepatitis in children: a multicenter clinicopathological study. Hepatology 2009;50:1113-20.

26. Shannon A, Alkhouri N, Carter-Kent C, Monti L, Devito R, Lopez R, et al. Ultrasonographic quantitative estimation of hepatic steatosis in children with NAFLD. J Pediatr Gastroenterol Nutr 2011;53:1905.

27. Park SE, Yang HR, Chang JY, Ko JS, Seo JK, Lee W, et al. Correlation of body mass index, body fat distribution, aminotranferases and computed tomography in obese children with fatty liver. Korean J Pediatr 2005;48:276-83.

28. Park SH, Kim PN, Kim KW, Lee SW, Yoon SE, Park SW, et al. Macrovesicular hepatic steatosis in living liver donors: use of CT for quantitative and qualitative assessment. Radiology 2006;239: 105-12.

29. Pacifico L, Martino MD, Catalano C, Panebianco V, Bezzi M, Anania C, et al. T1-weighted dual-echo MRI for fat quantification in pediatric nonalcoholic fatty liver disease. World J Gastroenterol 2011;17:3012-9.

30. Charatcharoenwitthaya P, Lindor KD. Role of radiologic modalities in the management of non-alcoholic steatohepatitis. Clin Liver Dis 2007;11:37-54.

31. Yoneda M, Yoneda M, Mawatari H, Fujita K, Endo H, Iida H, et al. Noninvasive assessment of liver fibrosis by measurement of stiffness in patients with nonalcoholic fatty liver disease (NAFLD). Dig Liver Dis 2008;40:371-8.

32. Nobili V, Vizzutti F, Arena U, Abraldes JG, Marra F, Pietrobattista A, et al. Accuracy and reproducibility of transient elastography for the diagnosis of fibrosis in pediatric nonalcoholic steatohepatitis. Hepatology 2008;48:442-8.

33. Chen J, Talwalkar JA, Yin M, Glaser KJ, Sanderson S0, Ehman
RL. Early detection of nonalcoholic steatohepatitis in patients with nonalcoholic fatty liver disease by using MR elastography. Radiology 2011;259:749-56.

34. Binkovitz LA, El-Youssef M, Glaser KJ, Yin M, Binkovitz AK, Ehman RL. Pediatric MR elastography of hepatic fibrosis: principles, technique and early clinical experience. Pediatr Radiol 2012;42:402-9.

35. Georgoff P, Thomasson D, Louie A, Fleischman E, Dutcher L, Mani H, et al. Hydrogen-1 MR spectroscopy for measurement and diagnosis of hepatic steatosis. AJR Am J Roentgenol 2012;199:27.

36. Vajro P, Lenta S, Socha P, Dhawan A, McKiernan P, Baumann U, et al. Diagnosis of nonalcoholic fatty liver disease in children and adolescents: position paper of the ESPGHAN Hepatology Committee. J Pediatr Gastroenterol Nutr 2012;54:700-13.

37. Manco M, Marcellini M, Giannone G, Nobili V. Correlation of serum TNF-alpha levels and histologic liver injury scores in pediatric nonalcoholic fatty liver disease. Am J Clin Pathol 2007;127:95460 .

38. Yang HR, Ko JS, Seo JK. Role of tumor necrosis factor-alpha promoter polymorphism and insulin resistance in the development of non-alcoholic fatty liver disease in obese children. Pediatr Gastroenterol Hepatol Nutr 2012;15:44-51.

39. Lebensztejn DM, Wojtkowska M, Skiba E, Werpachowska I, Tobolczyk J, Kaczmarski M. Serum concentration of adiponectin, leptin and resistin in obese children with non-alcoholic fatty liver disease. Adv Med Sci 2009;54:177-82.

40. Yang HR, Ko JS, Seo JK. The role of adipokines in the pathogenesis of non-alcoholic fatty liver disease in obese children; the relationship between body fat distribution and insulin resistance. Korean J Pediatr Gastroenterol Nutr 2007;10:185-92.

41. Louthan MV, Barve S, McClain CJ, Joshi-Barve S. Decreased serum adiponectin: an early event in pediatric nonalcoholic fatty liver disease. J Pediatr 2005;147:835-8.

42. Day CP, James OF. Steatohepatitis: a tale of two "hits"? Gastroenterology 1998;114:842-5.

43. Bell LN, Molleston JP, Morton MJ, Klipsch A, Saxena R, Vuppalanchi R, et al. Hepatic lipid peroxidation and cytochrome P-450 $2 \mathrm{E} 1 \mathrm{in}$ pediatric nonalcoholic fatty liver disease and its subtypes. J Clin Gastroenterol 2011;45:800-7.

44. Piemonte F, Petrini S, Gaeta LM, Tozzi G, Bertini E, Devito R, et al. Protein glutathionylation increases in the liver of patients with non-alcoholic fatty liver disease. J Gastroenterol Hepatol 2008;23 (8 Pt 2):e457-64.

45. Nobili V, Parola M, Alisi A, Marra F, Piemonte F, Mombello C, et al. Oxidative stress parameters in paediatric non-alcoholic fatty liver disease. Int J Mol Med 2010;26:471-6.

46. Bonnefont-Rousselot D, Ratziu V, Giral P, Charlotte F, Beucler I, Poynard T, et al. Blood oxidative stress markers are unreliable markers of hepatic steatosis. Aliment Pharmacol Ther 2006;23:91-8.

47. Wieckowska A, Zein NN, Yerian LM, Lopez AR, McCullough AJ, Feldstein AE. In vivo assessment of liver cell apoptosis as a novel biomarker of disease severity in nonalcoholic fatty liver disease. Hepatology 2006;44:27-33.

48. Yilmaz Y, Kedrah AE, Ozdogan 0. Cytokeratin-18 fragments and biomarkers of the metabolic syndrome in nonalcoholic steatohepatitis. World J Gastroenterol 2009;15:4387-91.

49. Vos MB, Barve S, Joshi-Barve S, Carew JD, Whitington PF, McClain CJ. Cytokeratin 18, a marker of cell death, is increased in children with suspected nonalcoholic fatty liver disease. J Pediatr Gastroenterol Nutr 2008;47:481-5.

50. Fitzpatrick E, Mitry RR, Quaglia A, Hussain MJ, DeBruyne R, 
Dhawan A. Serum levels of CK18 M30 and leptin are useful predictors of steatohepatitis and fibrosis in paediatric NAFLD. J Pediatr Gastroenterol Nutr 2010;51:500-6.

51. Sepulveda-Flores RN, Vera-Cabrera L, Flores-Gutierrez JP, Maldonado-Garza H, Salinas-Garza R, Zorrilla-Blanco P, et al. Obesityrelated non-alcoholic steatohepatitis and TGF-beta1 serum levels in relation to morbid obesity. Ann Hepatol 2002;1:36-9.

52. Miele L, Forgione A, La Torre G, Vero V, Cefalo C, Racco S, et al. Serum levels of hyaluronic acid and tissue metalloproteinase inhibitor-1 combined with age predict the presence of nonalcoholic steatohepatitis in a pilot cohort of subjects with nonalcoholic fatty liver disease. Transl Res 2009;154:194-201.

53. Lesmana CR, Hasan I, Budihusodo U, Gani RA, Krisnuhoni E, Akbar N, et al. Diagnostic value of a group of biochemical markers of liver fibrosis in patients with non-alcoholic steatohepatitis. J Dig Dis 2009;10:201-6.

54. Loaeza-del-Castillo A, Paz-Pineda F, Oviedo-Cardenas E, SanchezAvila F, Vargas-Vorackova F. AST to platelet ratio index (APRI) for the noninvasive evaluation of liver fibrosis. Ann Hepatol 2008; 7:350-7.
55. McGoogan KE, Smith PB, Choi SS, Berman W, Jhaveri R. Performance of the AST-to-platelet ratio index as a noninvasive marker of fibrosis in pediatric patients with chronic viral hepatitis. J Pediatr Gastroenterol Nutr 2010;50:344-6.

56. Ratziu V, Massard J, Charlotte F, Messous D, Imbert-Bismut F, Bonyhay L, et al. Diagnostic value of biochemical markers (FibroTest-FibroSURE) for the prediction of liver fibrosis in patients with non-alcoholic fatty liver disease. BMC Gastroenterol 2006;6:6.

57. Shah AG, Lydecker A, Murray K, Tetri BN, Contos MJ, Sanyal AJ, et al. Comparison of noninvasive markers of fibrosis in patients with nonalcoholic fatty liver disease. Clin Gastroenterol Hepatol 2009;7:1104-12.

58. Friedrich-Rust M, Rosenberg W, Parkes J, Herrmann E, Zeuzem S, Sarrazin C. Comparison of ELF, FibroTest and FibroScan for the non-invasive assessment of liver fibrosis. BMC Gastroenterol 2010;10:103.

59. Alkhouri N, Carter-Kent C, Lopez R, Rosenberg WM, Pinzani M, Bedogni G, et al. A combination of the pediatric NAFLD fibrosis index and enhanced liver fibrosis test identifies children with fibrosis. Clin Gastroenterol Hepatol 2011;9:150-5. 